\title{
Chest radiography and pneumonia in primary care: diagnostic yield and consequences for patient management
}

\author{
A.M. Speets*, A.W. Hoes", Y. van der Graaf*, S. Kalmijn\#, \\ A.P.E. Sachs ${ }^{\#}$ and W.P.Th.M. Mali*
}

ABSTRACT: The current prospective cohort study assessed the diagnostic yield of chest radiography (CXR) in primary-care patients suspected of pneumonia.

In total, 192 patients with a clinical suspicion of pneumonia aged $\geqslant 18$ yrs were referred by their general practitioner (GP) for CXR to one of the three participating hospitals in the Netherlands. All GPs were asked to complete a standardised form before and after CXR.

Pneumonia was diagnosed by GPs in 35 (18\%) patients, of whom 27 (14\%) patients had a positive CXR, and eight (4\%) patients a negative CXR, but with an assumed high probability of pneumonia by the GP. CXR clearly influenced the diagnosis of pneumonia by the GP in $53 \%$ of the patients. CXR ruled out pneumonia in $47 \%$ and the probability of pneumonia substantially increased in $6 \%$ of the patients. Patient management changed after CXR in $69 \%$ of the patients, mainly caused by a reduction in medication prescription (from 43 to $17 \%$ ) and more frequent reassurance of the patient (from 8 to $35 \%$ ).

In conclusion, pneumonia was frequently over diagnosed clinically by general practitioners. Chest radiography is a valuable diagnostic tool to substantially reduce the number of patients misdiagnosed and is particularly important for the exclusion of pneumonia in general practice.

KEYWORDS: Chest radiography, general practice, patient-care management, pneumonia

P rimary-care physicians usually rely on patient history, and signs and symptoms to diagnose or exclude pneumonia [1]. However, most signs and symptoms traditionally associated with pneumonia (e.g. fever and coughing) are not predictive of pneumonia in general practice [2-4]. Chest radiography (CXR) is the most frequently performed diagnostic investigation requested by general practitioners (GPs) in Europe. In $22 \%$ of patients with a suspected lower respiratory tract infection CXR is requested [5]. CXR is considered the gold standard for pneumonia diagnosis. CXR can diagnose pneumonia in cases with the presence of an infiltrate and differentiate pneumonia from other conditions that may present with similar symptoms (e.g. acute bronchitis). In addition, the results may suggest specific aetiologies (e.g. lung abscess), identify coexisting conditions (e.g. bronchial obstruction) and evaluate the severity of illness [6-9].

Although CXR is frequently used for diagnosing pneumonia, little is known about the influence of CXR on the probability estimation of pneumonia by GPs and on change in patient management. SIMPSON et al. [10] concluded that results of CXR requested by GPs influenced patient management in $48 \%$ of 97 patients with radiographical features of acute infection. However, the study by SIMPSON et al. [10] was only conducted in patients with radiographical evidence of infection and patient management was assessed via questionnaires filled in retrospectively by GPs. When assessing the diagnostic yield of CXR, e.g. in terms of patient management, it is important to study the complete cohort of patients suspected of pneumonia and not only the subgroup of patients with a radiographical diagnosis of pneumonia.

The objective of the current prospective cohort study was to assess: the effect of CXR on the probability estimation of pneumonia by GPs; the influence of CXR on patient management; and the consequences of CXR according to the patient. The study population consisted of primary-care patients with a clinical suspicion of pneumonia referred for CXR by GPs.

\section{METHODS AND MATERIALS}

\section{Study subjects}

The present study is part of a large prospective cohort study conducted from April 2003 to
AFFILIATIONS

*Dept of Radiology, and

\# Julius Centre for Health Sciences and Primary Care, University Medical Centre Utrecht, Utrecht, The Netherlands.

CORRESPONDENCE

A.M. Speets

Dept of Radiology (E01.335) University Medical Centre Utrecht P.0. Box 85500

3508 GA Utrecht The Netherlands Fax: 31302581098 E-mail: aspeets@umcutrecht.nl

Received:

January 202006

Accepted after revision:

June 292006 
December 2004 with the help of 78 GPs participating in the catchment area of one of three general hospitals located in three main cities in the Netherlands (Jeroen Bosch Hospital, 'sHertogenbosch; Gelre Hospitals, Apeldoorn; Onze Lieve Vrouwe Gasthuis, Amsterdam). In total, 870 patients aged $\geqslant 18$ yrs who were referred for CXR (postero-anterior and lateral view) by their GP to one of these hospitals were included in the cohort study. The study was approved by the medical ethics review board.

The GPs could fill in three probable diagnoses on a standard form before requesting a CXR. In the present study, all patients who were referred for CXR with a clinical suspicion of pneumonia as one of these probable diagnoses were included $(n=222)$. Thus, not all patients suspected of pneumonia were referred for CXR and included in the study. Only the patients in whom history and physical examination provided insufficient information for the GP to distinguish those with pneumonia from those without were included. Estimated probabilities for $18(8 \%)$ patients were not filled in by the GP before and/or after CXR. These patients were excluded from the study. Their patient characteristics were comparable with the included patients. Patients referred for a follow-up CXR for the treatment evaluation of pneumonia were also excluded $(n=12)$, resulting in a study population of 192 patients. Additionally, all patients with incidental pneumonia detected with CXR were included as a separate patient group (i.e. patients referred for CXR without a clinical suspicion of pneumonia).

\section{Methods}

All GPs were asked to complete a standardised form before requesting a $\mathrm{CXR}$, including information on: history; physical examination; indication; probable diagnosis with estimated prior probabilities on a visual analogue scale (range 0-100\%); and anticipated patient management. Abnormalities found during auscultation included crackles, rhonchi and/or bronchial breathing. Percussion was considered abnormal when dull or hyperresonant sounds were detected by the GP. The management options included: 1) referral to a medical specialist; 2) medication prescription; 3) reassurance of the patient; and 4) follow-up by the GP (watchful waiting or additional diagnostic testing). After the GP had requested a CXR, the patient could be referred for CXR to the general hospital the same day. In general, all CXRs were reported by a radiologist within $24 \mathrm{~h}$. Any significant abnormalities would be verbally reported to the GP, before the official radiological report was sent by mail. Therefore, significant abnormalities would normally be received by GPs within a day and the patient management plan could be adjusted directly. When no significant pathology is detected with CXR, it can take up to 4 days before the GP receives the official radiological report. Once the GP receives the report they complete a second questionnaire, again including the probable diagnosis with estimated posterior probabilities, and anticipated patient management plan. The current authors considered a decrease or increase in the estimated probability of pneumonia by the GPs after CXR of $\geqslant 30 \%$ as a substantial change in the probability estimation.

The findings on the CXR were categorised into four groups: 1) pneumonia; 2) other clinically relevant abnormalities; 3) a known abnormality, which was detected previously on CXR; and 4) no abnormality. Pneumonia was defined as a consolidation or infiltrate described by the radiologist in the CXR report, often summarised as pneumonia in the conclusion of the radiologist. A short questionnaire was sent to all patients 6 months after CXR (response rate 84\%), in order to evaluate current complaints and assess the consequences of CXR according to the patient.

\section{Analysis}

The primary outcome measures for the present study were the proportion of patients with a clear shift in the probability estimation of pneumonia by the GP $(\geqslant 30 \%$ decrease or increase of the estimated probability after CXR), and the proportion of patients in whom there was a change in patient management by the GP following CXR.

\section{RESULTS}

The mean age of the patients with a clinical suspicion of pneumonia was $56.8 \pm 17.6$ yrs and $55 \%$ were male. In total, $15 \%$ of the patients had a prior diagnosis of pneumonia. Cough was the most frequently reported symptom among the patients $(66 \%)$. Abnormalities during auscultation and percussion were found in 59 and $26 \%$ of the patients, respectively (table 1).

The radiology reports of CXR showed: pneumonia in 27 (14\%) patients; other clinically relevant abnormalities in 32 (17\%) patients; a known abnormality, which was detected previously on CXR, in 35 (19\%) patients; and no abnormality in 98 (52\%) patients. The group of patients with other clinically relevant abnormalities consisted of one patient with malignancy, 23 patients with chronic obstructive pulmonary disease (COPD)/

\begin{tabular}{lc} 
TABLE 1 Patient characteristics & \\
Subjects n & 192 \\
Age yrs & $56.8 \pm 17.6$ \\
Sex male & $106(55)$ \\
Prior diagnoses & \\
$\quad$ Malignancy & \\
Pneumonia & $9(5)$ \\
COPD/asthma/chronic bronchitis & $28(15)$ \\
Recent prescription of antibiotics & $48(25)$ \\
History & $45(23)$ \\
Smoking & \\
Pain & $32(17)$ \\
Haemoptysis & $27(14)$ \\
Cough & $13(7)$ \\
Dyspnoea & $127(66)$ \\
Other symptoms of respiratory infection & $54(28)$ \\
Fever & $39(20)$ \\
General malaise & $33(17)$ \\
Physical examination & $25(13)$ \\
Abnormalities during auscultation & \\
Abnormalities during percussion & $113(59)$ \\
\hline
\end{tabular}

Data are presented as mean \pm SD or $n(\%)$, unless otherwise stated. COPD chronic obstructive pulmonary disease. " : Various locations $n=7$, lung $n=2$; abnormal sputum, nasal congestion, throat symptoms and complaints of a cold 
asthma/chronic bronchitis, four with abnormalities that required further investigation and four patients with other abnormalities (e.g. diaphragmatic hernia).

The distributions of the prior and posterior probability of pneumonia are shown in figure 1 . The number of patients with a low $(<30 \%)$, moderate $(30-70 \%)$ or high $(>70 \%)$ probability of pneumonia according to the GP before and after CXR are shown in figure 2. Most noticeable were the two large groups referred for CXR with a very low or high prior probability of pneumonia, $64(33 \%)$ patients and $30(16 \%)$ patients, respectively. After CXR, pneumonia was diagnosed in four out of the $64(6 \%)$ patients with a very low prior probability and in only 15 out of the $30(50 \%)$ patients with a very high prior probability of pneumonia. The probability estimation of pneumonia was clearly changed by means of CXR in $53 \%$ of the patients (95\% confidence interval (CI) 46-59\%). The estimated probability of pneumonia decreased with $\geqslant 30 \%$ (range $30-100 \%$ ) in $89(47 \%$ ) patients and increased with $\geqslant 30 \%$ (range $30-80 \%$ ) in $12(6 \%)$ patients after CXR.

The proportion of patients for whom patient management changed following CXR was 69\% (95\% CI 62-75\%). Main changes in patient management plans after CXR included: 1) a reduction in the number of patients with a medication
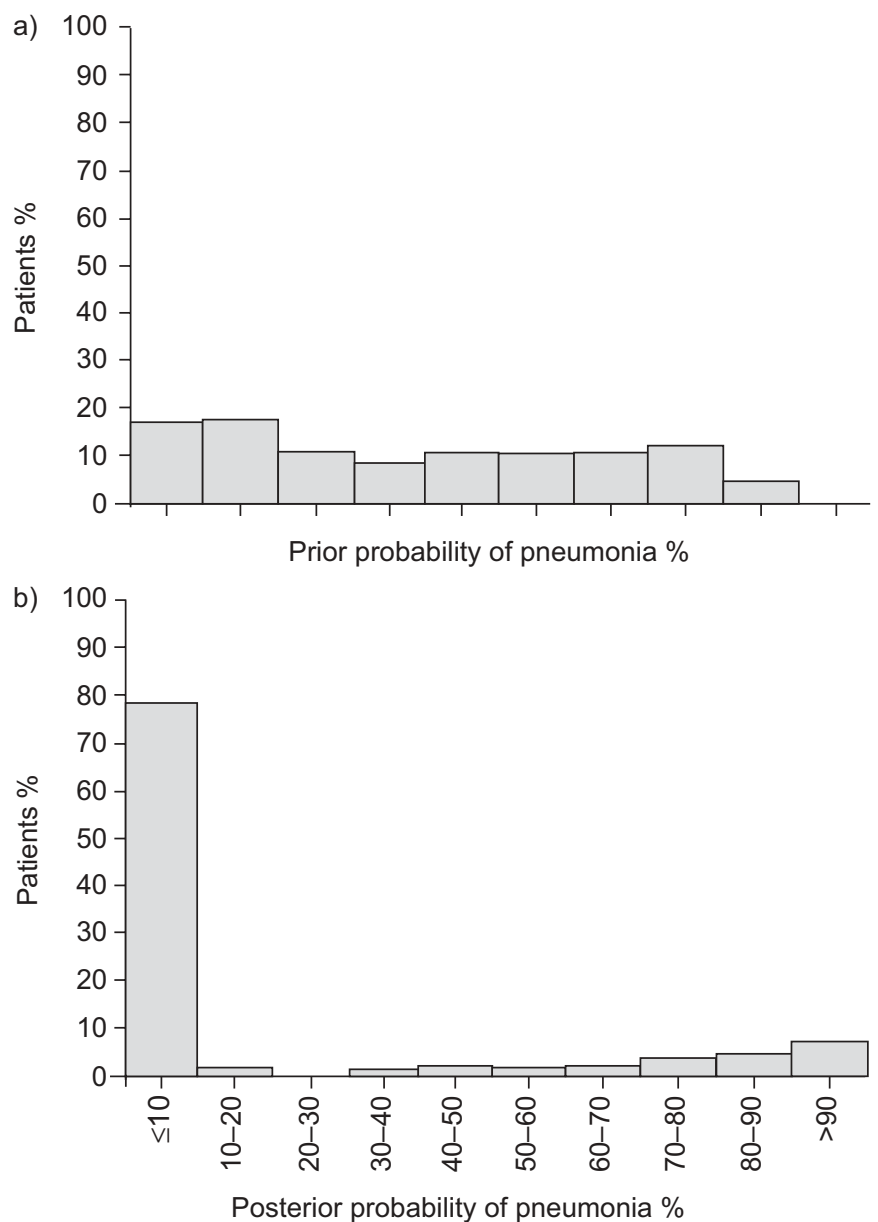

FIGURE 1. Distribution of the probabilities of pneumonia estimated by the general practitioners a) before and b) after chest radiography. prescription from $79-32$ (43-17\%) patients; and 2) more frequent reassurance of the patient, from 15-64 (8-35\%) patients (table 2). The reduction in medication prescription was mainly caused by a decrease in the prescription of antibiotics from $53(28 \%)$ patients before CXR to $26(14 \%)$ patients after CXR. The current complaints were diminished or disappeared in almost $80 \%$ of the patients referred for CXR by GPs with a clinical suspicion of pneumonia 6 months after CXR. Only $15 \%$ of the patients who returned the questionnaire reported that CXR had no value. CXR resulted in a definite diagnosis or better treatment according to $43 \%$ of the patients, and $44 \%$ of the patients were reassured after CXR.

Pneumonia was diagnosed with CXR in 27 (14\%) patients, with a mean age of $53.8 \pm 18.8 \mathrm{yrs}$, and $44 \%$ were male. Abnormalities during auscultation and percussion were found in 74 and $26 \%$ of these patients, respectively. The GPs referred seven $(26 \%)$ patients to a medical specialist, medications were prescribed in $13(48 \%)$ patients, patient management was watchful waiting in six (22\%) patients, and an additional computed tomography scan was ordered for one (4\%) patient. The current complaints were diminished or disappeared 6 months after CXR in $72 \%$ of the patients, and $8 \%$ reported that CXR had no value for them.

Additionally, pneumonia was diagnosed by the GP in eight (4\%) patients without a positive CXR, but with an assumed high probability of pneumonia by the GP. The GP suspected pneumonia in four patients, viral pneumonia in two, and mycoplasma pneumonia was shown with additional laboratory investigation in two patients. The four patients suspected of pneumonia were: 1) a 48-yr-old male with a medical history of COPD, with a 2-week complaint of cough and thoracic pain, and without abnormalities during physical examination; 2) a 52-yr-old female who smoked, with a 1-week complaint of cough, dyspnoea and fever, and without abnormalities during physical examination; 3) a 62-yr-old female with a colleague diagnosed with pneumonia, a 1.5-week complaint of cough, and crepitations on the left side; and 4) a 20-yr-old female with an infiltrate in her medical history ( 2.5 yrs previously), some days complaint of cough, thoracic pain and fever, and without abnormalities during physical examination. After CXR, four out of the eight patients were referred to a medical specialist and medications were prescribed in four patients. The current complaints were diminished or disappeared in $71 \%$ of the patients 6 months after CXR and 14\% reported that CXR had no value for them.

Small infiltrates or early manifestations of pneumonia were found as an incidental finding with CXR in five patients (age range 32-77 yrs; three males) of the total cohort of $870(<1 \%)$ patients. Two patients were referred for CXR for the exclusion of a malignancy, one patient for the confirmation of COPD, and two patients had unclear complaints without any abnormalities during physical examination. After CXR three patients were referred to a medical specialist, medications were prescribed to one patient, and patient management was watchful waiting and an additional follow-up CXR for one patient.

\section{DISCUSSION}

CXR clearly influenced the diagnosis of pneumonia by the GP in $56 \%$ of the patients referred for CXR with a clinical suspicion 


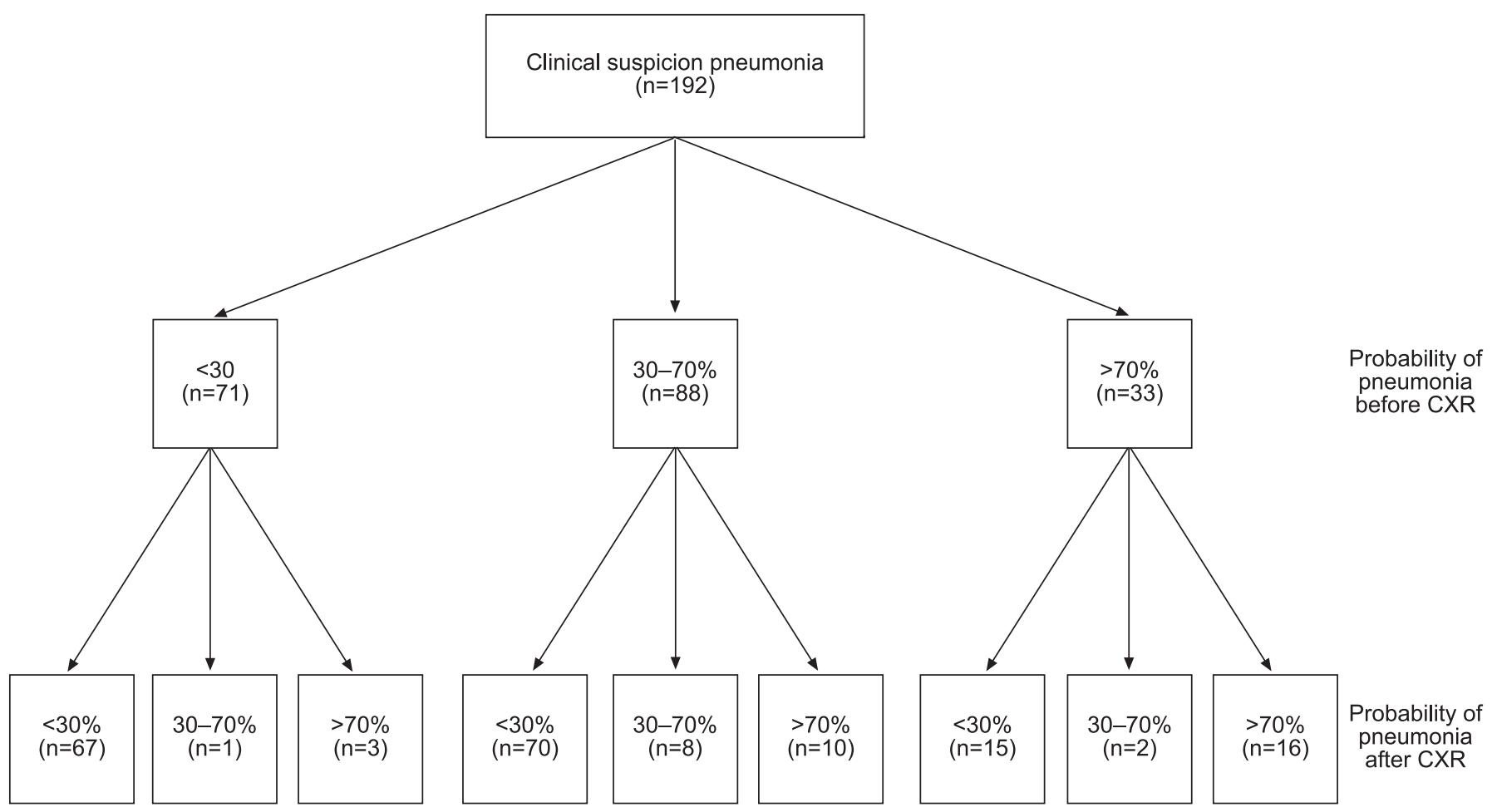

FIGURE 2. Flow diagram showing the number of patients with a low $(<30 \%)$, moderate $(30-70 \%)$ or high $(>70 \%)$ probability of pneumonia according to the general practitioner before and after chest radiography (CXR).

of pneumonia: CXR ruled out pneumonia in 50\% of the patients, and the probability of the diagnosis of pneumonia substantially increased in $6 \%$ of the patients. The proportion of patients for whom patient management changed following CXR was $69 \%$, mainly caused by a decrease in the prescription of antibiotics and more frequent reassurance of the patient.

To the present authors' knowledge, the current study is the first that has assessed the effect of CXR on the probability estimation of pneumonia by GPs. The number of patients in whom patient management changed $(69 \%)$ is much higher than the $48 \%$ reported in the study of SIMPSON et al. [10]. This difference could be explained by the study designs. The study by SIMPSON et al. [10] was conducted in patients with radiographical evidence of infection and patient management was assessed with questionnaires that were filled in retrospectively by GPs, which may have biased the results. Besides, SIMPSON et al. [10] did not specify whether reassurance of the patient was considered as patient management and how patient management was influenced by the findings of CXR.

The distributions of the prior and posterior probability of pneumonia in figure 1 show that the uncertain area of a diagnosis, around estimated probabilities of $50 \%$, disappeared

\section{TABLE 2 Patient management plans of general practitioners (GPs) before and after chest radiography ${ }^{\#}$}

\begin{tabular}{lllll} 
& Before & & \\
\hline $\begin{array}{c}\text { Referral medical } \\
\text { specialist }\end{array}$ & Medication prescription & Reassurance & Follow-up by GP & Total
\end{tabular}

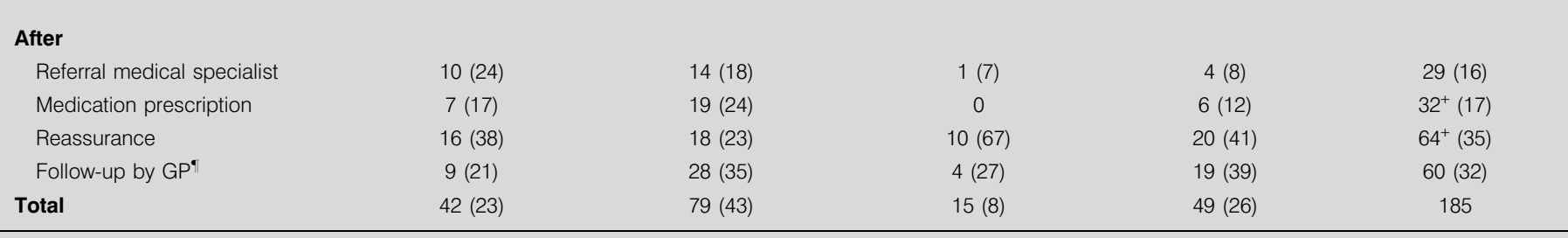

Data are presented as $\mathrm{n}$ or $\mathrm{n}(\%) .{ }^{*}$ : Patient management plans for seven (4\%) patients were not filled in by the GP before and/or after chest radiography; ${ }^{\circ}$ : follow-up by GP, predominantly watchful waiting or additional diagnostic testing, such as spirometry or laboratory investigation; ${ }^{+}$: the differences in proportions of patient management after chest radiography were significant with a p-value $\leqslant 0.05$. 
largely as a consequence of CXR. Most noticeable in the current study was that almost half of all patients were referred for CXR with a very low or high prior probability of pneumonia, 33\% and $16 \%$ of the patients, respectively. In $75 \%$ of the patients with a very low prior probability of pneumonia the GPs had additional differential diagnoses, such as COPD or acute bronchitis. After CXR, pneumonia was diagnosed in only $6 \%$ of the patients with a very low prior clinical probability of pneumonia, and therefore, CXR was not a useful tool for diagnosing pneumonia in these patients. Pneumonia was diagnosed after CXR in only $50 \%$ of the patients with a very high prior probability of pneumonia. This emphasises the importance of referring patients with a clinical suspicion of pneumonia for CXR, even when the prior probability of pneumonia is very high according to the GP.

Pneumonia was diagnosed by the GP in $35(18 \%)$ patients, of whom $27(14 \%)$ had a positive and eight (4\%) patients a negative CXR but an assumed high probability of pneumonia by the GP. Low percentages of patients diagnosed with pneumonia by a positive CXR were also found in other studies: $15 \%$ by MELBYE et al. [11], and $7 \%$ by LIEBERMAN et al. [12]. It is noticeable that the estimated probabilities in the patient groups diagnosed with pneumonia with a positive and negative CXR were high before CXR, 61 and $72 \%$ respectively. However, these percentages were not high enough for the GPs to start treatment or refer patients to a medical specialist without an additional CXR. The current restrictive policy of prescribing antibiotics could encourage GPs to order CXR in patients suspected of pneumonia even when estimated prior probabilities are high based on medical history, anamnesis and physical examination [9].

The manifestations of pneumonia on CXR may vary considerably, depending upon the degree of inflammation and the stage of the disease process. It is difficult to diagnose mild or early stage pneumonia by CXR $[13,14]$. However, it is possible to detect pneumonia during physical examination without radiographical evidence [13]. The eight patients with a high estimated probability of pneumonia and a negative CXR might have been referred too soon for CXR by their GP; mycoplasma pneumonia was shown with additional laboratory investigation in two out of the eight patients.

Interestingly, no clear differences in patient characteristics, including signs and symptoms, were observed in referred patients with or without pneumonia. This indicates that the GPs adequately applied clinical skills to select those patients for additional imaging in whom history and physical examination provided insufficient information to distinguish those with pneumonia from those without.

As expected, incidental findings of pneumonia were scarce with CXR. In the current study, small infiltrates or early manifestations of pneumonia were incidental findings in $<1 \%$ of the total cohort of 870 patients.

A limitation of the present study was that it was impossible to verify whether or not the GP really would have conducted the anticipated patient management in accordance with the plan made on the standardised form before CXR was performed. This could result in an overestimation of intended referrals to medical specialists.
In conclusion, pneumonia was frequently over diagnosed clinically by the general practitioners in the current study. Chest radiography is a valuable diagnostic tool in primary-care patients with a clinical suspicion of pneumonia who are referred for chest radiography to substantially reduce the number of patients misdiagnosed. In particular, chest radiography was important for the exclusion of pneumonia in general practice. Chest radiography was not very useful for diagnosing pneumonia in patients with a low clinical probability of pneumonia.

\section{ACKNOWLEDGEMENTS}

It would not have been possible to conduct this study without the participation of all general practitioners from the catchment areas of the three hospitals. The authors would like to thank the radiologists and trial nurses for their help with all the logistics in the three participating hospitals: M. Rutten and H. de Koning (Jeroen Bosch Hospital, 's-Hertogenbosch); J.W. Gratama and I. Brussee (Gelre Hospitals, Apeldoorn); and A. Montauban van Swijndregt and C. Kressenhof (Onze Lieve Vrouwe Gasthuis, Amsterdam). The authors would also like to thank C. Haaring from the University Medical Centre Utrecht for creating the database and assisting with the data management.

\section{REFERENCES}

1 Metlay JP, Fine MJ. Testing strategies in the initial management of patients with community-acquired pneumonia. Ann Intern Med 2003; 138: 109-118.

2 Hopstaken RM, Muris JW, Knottnerus JA, Kester AD, Rinkens PE, Dinant GJ. Contributions of symptoms, signs, erythrocyte sedimentation rate, and C-reactive protein to a diagnosis of pneumonia in acute lower respiratory tract infection. Br J Gen Pract 2003; 53: 358-364.

3 Metlay JP, Kapoor WN, Fine MJ. Does this patient have community-acquired pneumonia? Diagnosing pneumonia by history and physical examination. JAMA 1997; 278: 1440-1445.

4 Mabie M, Wunderink RG. Use and limitations of clinical and radiologic diagnosis of pneumonia. Semin Respir Infect 2003; 18: 72-79.

5 Woodhead M, Gialdroni Grassi G, Huchon GJ, Leophonte P, Manresa F, Schaberg T. Use of investigations in lower respiratory tract infection in the community: a European survey. Eur Respir J 1996; 9: 1596-1600.

6 Niederman MS, Mandell LA, Anzueto A, et al. American Thoracic Society. Guidelines for the management of adults with community-acquired pneumonia. Diagnosis, assessment of severity, antimicrobial therapy, and prevention. Am J Respir Crit Care Med 2001; 163: 1730-1754.

7 British Thoracic Society Standards of Care Committee, BTS Guidelines for the management of community acquired pneumonia in adults. Thorax 2001; 56: Suppl. 4, IV1-IV64.

8 Mandell LA, Marrie TJ, Grossman RF, Chow AW, Hyland RH. Canadian guidelines for the initial management of community-acquired pneumonia: an evidencebased update by the Canadian Infectious Diseases Society and the Canadian Thoracic Society. The Canadian Community-Acquired Pneumonia Working Group. Clin Infect Dis 2000; 31: 383-421. 
9 Bartlett JG, Dowell SF, Mandell LA, File TM Jr, Musher DM, Fine MJ. Practice guidelines for the management of community-acquired pneumonia in adults. Infectious Diseases Society of America. Clin Infect Dis 2000; 31: 347-382.

10 Simpson JCG, Hulse P, Taylor PM, Woodhead M. Do radiographic features of acute infection influence management of lower respiratory tract infections in the community? Eur Respir J 1998; 12: 1384-1387.

11 Melbye H, Straume B, Aasebo U, Brox J. The diagnosis of adult pneumonia in general practice. The diagnostic value of history, physical examination and some blood tests. Scand J Prim Health Care 1988; 6: 111-117.

12 Lieberman D, Shvartzman P, Korsonsky I, Lieberman D. Diagnosis of ambulatory community-acquired pneumonia. Comparison of clinical assessment versus chest X-ray. Scand J Prim Health Care 2003; 21: 57-60.

13 Fraser RG, Paré JAP, eds, Pneumonia. Diagnosis of diseases of the chest. 2nd Edn. Philadelphia, W.B. Saunders Company, 1978.

14 Melbye H. Community pneumonia - more help is needed to diagnose and assess severity. Br J Gen Pract 2002; 52: 886-888. 